index ecomunicación | no 12(1) 2022 | Páginas 261-284

E-ISSN: 2174-1859 | ISSN: 2444-3239 | Depósito Legal: M-19965-2015

Recibido el 26_05_2021 | Aceptado el 21_09_2021 | Publicado el 15_01_2022

\title{
SEXO, DROGAS Y SERIES DE ADOLESCENTES: ANÁLISIS DE LAS ACTITUDES SOCIALES DE LOS ADOLESCENTES EN LAS SERIES TELEVISIVAS
}

SEX, DRUGS AND TEEN-SERIES: BEHAVIORAL ANALYSIS OF THE TEEN CHARACTERS IN TV-SHOWS

https://doi.org/10.33732/ixc/12/01Sexodr

Mayte Donstrup

Universidad de Sevilla mdonstrup@us.es https://orcid.org/0000-0001-6236-4967 
Resumen: El propósito de este trabajo es analizar las actitudes y las conductas sociales de los protagonistas de las teen series, es decir, de las series de ficción dirigidas a adolescentes. Con este fin se ha realizado un análisis de contenido, técnica que ha permitido identificar los comportamientos y las consecuencias más habituales en dichas series de ficción. El universo de estudio se ha compuesto de la primera temporada de las siete series mejor valoradas por su público en el primer trimestre de 2020. En total, la muestra ha resultado en siete series de televisión: Élite (Netflix: 2018- ), Chilling Adventures of Sabrina (Las escalofriantes aventuras de Sabrina, Netflix: 2018- ), Sex Education (Netflix: 2019- ), 13 Reasons Why (Por trece razones, Netflix: 2017), I Am Not Okay with This (Esta Mierda me supera, Netflix: 2020- ), Riverdale (The WC: 2017- ) y Euphoria (HBO: 2019- ). Los resultados indican un alto porcentaje de escenas de sexo sin protección y de consumo de alcohol sin apenas consecuencias negativas.

Palabras clave: ficción televisiva; series de televisión; adolescentes; alcohol; tabaco; sexo; drogas.

Abstract: The aim of this study is to analyze the attitudes and social behaviors of the protagonists of teen series; that is, fiction series produced for teenagers. With this aim, a content analysis has been carried out, a technique that has allowed the identification of the most common behaviors and consequences in said fiction series. The sample is composed of the first season of the seven series best rated by their audience in the first quarter of 2020: Elite (Netflix: 2018- ), Chilling Adventures of Sabrina (Netflix: 2018- ), Sex Education (Netflix: 2019- ), 13 Reasons Why (Netflix: 2017), I Am Not Okay with This (Netflix: 2020- ), Riverdale (The WC: 2017- ) y Euphoria (HBO: 2019- ). The results indicate a high percentage of scenes of unprotected sex and alcohol consumption with hardly any negative consequences.

Keywords: TV Fiction; TV Series; Teenagers; Alcohol; Tobacco; Sex; Drugs. 


\section{Introducción}

Las series de televisión constituyen interesantes objetos de estudio, pues a través de ellas podemos ver cómo se conceptualizan a los diferentes grupos sociales. En este sentido, tal como afirma Jager (2003), los discursos —entre los que se incluyen los ficcionales- son agentes creadores de conciencia a través de los cuales fluyen conocimientos - símbolos colectivos-que se insertan e interactúan en la sociedad. De hecho, el rol social de la televisión es una temática ampliamente estudiada en la literatura científica - por poner unos ejemplos, los trabajos de Jensen (1994), Castelló (2010), Dhaenens (2012) o Nærland (2020) - . De acuerdo con Thornham y Purvis, la ficción televisiva combina «un interés por las estructuras sociales y culturales en el modo en el que los individuos se representan a sí mismos en esas estructuras y dan sentido a la representación pública de las mismas» (2012: 76) ${ }^{1}$.

En la amplia variedad de temáticas que abordan las series de ficción, en este trabajo vamos a focalizar nuestra atención en las que se encuentran protagonizadas por personajes adolescentes; es decir, las teen series. Cabe señalar que las funciones sociales de la televisión adquieren un especial interés durante la adolescencia, pues esta etapa de desarrollo se considera crucial para el desarrollo de la identidad personal (Arnett, Larson y Offer, 1995: 513). En específico, nuestro principal objetivo será estudiar las temáticas que más llaman la atención de los adolescentes: el amor, el sexo y las relaciones íntimas (Masanet, 2016: 49). Así, examinaremos los «aspectos de la realidad que les rodea y de los que habitualmente no se habla, como las drogas, la sexualidad, la pobreza o los problemas graves que pueden vivir las familias» (Andrés, 2005: 83).

De este modo, en este trabajo se realizará, en primer lugar, un repaso por las características y problemáticas abordadas por las teen series para posteriormente analizar la representación del sexo y las drogas (legales e ilegales) en siete de las series de adolescentes más populares del primer trimestre del 2020: Élite (Netflix: 2018- en curso), Chilling Adventures of Sabrina (Las escalofriantes aventuras de Sabrina, Netflix: 2018-2020), Sex Education (Netflix: 2019-En curso), 13 Reasons Why (Por trece razones, Netflix: 2017-2020), I Am Not Okay with This (Esta Mierda me supera, Netflix: 2020), Riverdale (The WC: 2017-En curso ) y Euphoria (HBO: 2019-En curso).

\footnotetext{
${ }^{1}$ A concern with social and cultural structures with a concern for how individuals both represent those structures to themselves and make sense of public representation of them.
} 


\section{Series de televisión dirigidas a adolescentes}

Aunque no podamos considerar a las teen series como un género autónomo e independiente, sí que existen elementos que permiten caracterizar sus temáticas y enmarcarlas dentro de unos parámetros comunes (Fedele y García-Muñoz, 2010). Ante todo, las teen series se caracterizan por encontrarse dirigidas al público adolescente y, por ello, estar escenificadas sobre dos principales ejes: la familia y el instituto, con una primacía de las tramas centradas en las relaciones sociales fuera del hogar (Guarinos, 2009; García-Muñoz y Fedele, 2011; Lacalle, 2013). Por su parte, en el ámbito social, las relaciones sexuales y la amistad son dos de los pilares que sustentan las narrativas de las series dirigidas a adolescentes (Lacalle, 2013). En el ámbito privado, las temáticas más explotadas son la rebelión de los adolescentes, el cuestionamiento a la autoridad y el manejo de la incertidumbre (Falcón y Díaz-Aguado, 2014: 153). Respecto a los protagonistas adolescentes, estos se encuentran enmarcados en un particular arquetipo: «joven, atractivo y vestido a la moda, perteneciente a una uniforme clase media y media-alta» (Lacalle, 2013: 30).

Un aspecto que ocupa un considerable peso en la trama de las teen series son las primeras relaciones sentimentales, que tienden a representar un amor romántico basado en diferentes mitos, como el del 'amor verdadero predestinado', 'el poder del amor' o 'el amor como posesión' (Masanet, Medina-Bravo, y Aran-Ramspott, 2016). Además, en el manejo de la incertidumbre adolescente detectamos una autoexploración de la identidad (Falcón and Díaz-Aguado, 2014) donde se tocan los comportamientos y actitudes individuales o los roles de género. Al respecto de los cometidos asignados a los chicos y las chicas, las teen series se balancean entre el mantenimiento de los estereotipos de género (García-Muñoz y Fedele 2011; Masanet, Medina-Bravo, y Aran-Ramspott 2016; Signorielli 2007) o, en menor medida, el derrocamiento de estos (Banks 2004; Feasey 2006; Raya Bravo, 2019; Osgerby 2004; Owen 1999; Masanet y Fedele, 2019; Mateos-Pérez, 2021). En definitiva, las series dirigidas a adolescentes «son contenidos que buscan la autenticidad, historias y personajes ligados a la realidad [y que] emplean una jerga juvenil, reiterativa, espontánea, propia de la clase social que representan» (Mateos-Pérez, 2021: 154).

\subsection{Alcohol y tabaco en series juveniles}

En términos generales, el consumo de alcohol o la presencia de product placement de bebidas espirituosas se encuentran normalizados en la programación televisiva (Austin y Meili 1994; Christensen, Henriksen, y Roberts, 2000). Diferentes estudios han detectado en la ficción televisiva un alto consumo de alcohol (Christensen, Henriksen y Roberts, 2000; Russell, Dale y Cristell, 2008; March Cerdá, Martín Barato, López Doblas, Luque Martín, Prieto Rodríguez y 
Danet Danet, 2014) o referencias al mismo (Russell, 2006). Por regla general, las series de televisión tratan con frecuencia a la bebida como algo humorístico y la asocian con la camaradería (Hundley, 1995). En ciertas ocasiones, la bebida también se asocia con consecuencias negativas, como las relaciones sexuales insatisfactorias, la adicción, los accidentes o la muerte. Sin embargo, cuando el consumo de bebidas alcohólicas se asocia con resultados negativos suele ser combinándolos con rasgos positivos del suceso (Russell y Russell, 2009).

Al igual que en la programación adulta, en las series dirigidas a adolescentes nos encontramos con que el consumo de alcohol se encuentra normalizado en el tiempo dedicado al ocio; por ejemplo, en el estudio de 76 episodios de la popular serie estadounidense The OC (Fox: 2003-2007), Russell, Russell y Grube (2009) encontraron que el 89,5\% mostraron resultados positivos tras el consumo de bebidas espirituosas, mientras que el 60,5\% desarrollaron consecuencias indeseadas. No obstante, en términos excluyentes, solo el 2,9\% de los episodios mostraron resultados negativos (sin ningún aspecto positivo), mientras que el $34,3 \%$ de los episodios con alcohol mostraron exclusivamente los beneficios: «Aunque el consumo excesivo de alcohol se representa asiduamente en The $O C$, los resultados negativos para la salud fueron los mensajes menos representados» (2009: 101)2. Los mensajes más comunes sobre el alcohol son: la facilidad para sentirse relajado $(76,3 \%)$, la diversión $(35,5 \%)$, expresar sentimientos (25\%), felicidad (19.7\%), autoestima (18.4\%) y habilidades sociales $(14,5 \%)$. Los mensajes positivos sobre el consumo del alcohol no se limitan a las series estadounidenses; a modo de ilustración, un análisis de contenido sobre la programación francesa determinó que:

\begin{abstract}
Las series francesas codificadas representan el alcohol con más frecuencia, y de forma más positiva, que las series estadounidenses. El alcohol se asocia a los personajes principales con los que la audiencia puede identificarse. Además, los personajes asociados con el alcohol son en su mayoría deseables, con una buena situación profesional o personal, o ambas. Estas características aumentan las posibilidades de que los espectadores se identifiquen con el personaje y minimicen las consecuencias del consumo de sustancias (Chapoton, Werlen y Denois 2019: 4). ${ }^{3}$
\end{abstract}

\footnotetext{
${ }^{2}$ Although heavy drinking is regularly portrayed in The O.C., negative health outcomes were the least depicted messages.

3 French TV series show alcohol more often than the US TV series coded and in a more positive way. Alcohol is associated with the main characters with whom the audience can identify: within the sample coded, characters associated with alcohol are indeed mostly stable and desirable with good professional or personal situation or both. Such characteristics increase the viewers' chances of identifying with a character and minimizing consequences of substances consumption, raising in return the risk of identification to a fictional character and the use of substances.
} 
Respecto al tabaco, aunque su presencia audiovisual se encuentre más restringida, nos encontramos con series de televisión que ligan al carácter del personaje el hábito de fumar; por ejemplo, la pareja rebelde de Física o Química (Antena 3: 2008-2011) formada por Gorka y Ruth, o el atractivo y peligroso Duque de Sin Tetas no hay Paraíso (Telecinco: 2008-2011) (Reñones, Valdivieso López y Victoria Arijaa, 2011).

\subsection{Relaciones sexuales en series de adolescentes}

Otro tema que ha abarcado una considerable discusión académica es la representación del sexo en las series de adolescentes (Kellner, 1995), donde se entremezclan los estereotipos de género y actitudes más progresistas. Por norma general, los personajes tienen encuentros sexuales a una edad más temprana (Eyal, Kunkel, Biely y Finnerty, 2007) y se dan ocasiones en las que las relaciones sexuales se dan en relaciones espontáneas sin compromiso (Eyal, Kunkel, Biely y Finnerty, 2007) o en las que se requiere un compromiso posterior (Gunter, 1995). Además, las chicas suelen iniciar el diálogo sexual (Aubrey, 2004), pero los chicos dan el primer paso hacia el acto (Batchelor, Kitzinger y Burtney, 2004). Los personajes sexualmente activos rara vez toman precauciones contra el embarazo o las enfermedades de transmisión sexual (ETS) y con poca frecuencia experimentan consecuencias como resultado de sus acciones sexuales (Kunkel et. al. 2001; Sprafkin y Silverman, 1981; Tuglio, 1998; Aubrey, 2004; Hust, Brown y L'Engle, 2008; L'Engle, Brown, y Kenneavy, 2006; Wright, 2009). $\mathrm{Al}$ respecto, ellas son las únicas responsables de la anticoncepción, existiendo pocos ejemplos de adolescentes (varones) que manifiestan preocupaciones sobre el sexo seguro (Gavin, 2001; Batchelor et al., 2004; Van Damme y Van Bauwel, 2013).

En lo concerniente al consentimiento, las adolescentes limitan al ámbito privado su verbalización sobre estar preparadas para mantener relaciones sexuales. En caso de expresar sus deseos abiertamente son caracterizadas como 'malas chicas' (Aubrey, 2004; Bindig, 2008; Ortiz y Brooks, 2014). De hecho, ellas suelen ser degradadas como objetos sexuales (Bindig, 2008; Van Damme, 2010) por diferentes vías: las chicas usan sus cuerpos para seducir o intimidar a un chico para que haga algo; abuso de encuadres de áreas específicas de las mujeres como los pechos y los muslos; las chicas como trofeo por el que los chicos juegan durante un juego, o que se ofrecen por propia voluntad como recompensa para agradecer a un chico su esfuerzo (Van Damme, 2010).

Por su parte, la pérdida de la virginidad significa para los chicos un estatus social más alto y para las chicas una decisión moral (Kelly, 2010; Crowley Webber, 2019). Además, persiste el estereotipo del chico que tiene sexo con tantas chicas como puede, porque un 'hombre de verdad' nunca rechaza 
una oportunidad para mantener sexo (Brown et al., 2002; Kim et al., 2007; Signorielli, 2007; Bindig, 2008). En síntesis, el doble rasero hace que la sexualidad de los chicos sea animada y recompensada, con personajes masculinos retratados como activos en la elección (o incluso depredadores) en contraste con la pasividad, la restricción y el cumplimiento de la sexualidad femenina (Aubrey, 2004).

\section{Objetivos}

Una vez expuestas las principales características del consumo de alcohol y/o tabaco y las relaciones sexuales en las series de televisión dirigidas a adolescentes, se propone como principal objetivo de esta investigación el conocer los hábitos de los personajes adolescentes en las series más populares del primer semestre de 2020. A partir del mencionado objetivo general, los objetivos específicos son los siguientes:

0.1.1. Detectar la representación del consumo del alcohol y tabaco en las series televisivas dirigidas a adolescentes.

0.1.2. Identificar la representación de las relaciones sexuales entre los adolescentes protagonistas.

0.1.3. Detectar si existen diferencias de género en el comportamiento de los personajes según su sexo.

\section{Metodología}

Para alcanzar los objetivos propuestos se plantea un enfoque cuantitativo a través de la realización de un análisis de contenido (Krippendorf, 1990). De este modo, se ha diseñado una parrilla específica que permita cuantificar las escenas de sexo y de consumo de drogas, legales e ilegales, en las series de televisión de adolescentes, así como sus consecuencias. Asimismo, para el estudio narrativo se ha considerado bibliografía específica sobre el estudio de los personajes (Casetti y di Chio, 2010; Chatman, 2013); de esta forma, el análisis de los personajes tendrá en consideración su rango como persona, es decir, una personalidad compuesta de un rango de matices propios que constituyen su identidad (Casetti y di Chio, 2010).

\subsection{Diseño de la investigación}

Con el objeto de explotar los datos, se han adaptado las categorías establecidas por los estudios de Aubrey (2004), Russell, Russell y Grube (2009), Wright (2009), Reñones, Valdivieso López y Victoria Arijaa (2011) y Van Damme (2010). De este modo, se asociarán las acciones estudiadas con sus consecuencias y el carácter del personaje que las ejecuta. En la temática sexual, siguiendo a Aubrey (2004), las consecuencias negativas son conceptualizadas como los 
resultados indeseables, como pueden ser la condena al ostracismo o los embarazos indeseados (Wright, 2009; Van Damme, 2010). Así, las consecuencias negativas pueden tener raíces emocionales, físicas o punitivas (Aubrey, 2004). En lo concerniente al consumo de drogas, vamos a separar las drogas legales, como el alcohol y el tabaco, de las ilegales, como pueden ser la marihuana o la cocaína. Según los resultados de Russell, Russell y Grube (2009), los mensajes más comunes sobre el alcohol son: la facilidad para sentirse relajado, la diversión, el expresar sentimientos, relajación (felicidad, despreocupación, etc.), la autoestima y las habilidades sociales. Las consecuencias negativas pueden ser las relaciones sexuales lamentables, la adicción, los accidentes o la muerte (Russell y Russell, 2009). De acuerdo con los objetivos de nuestro estudio, en este trabajo vamos a aplicar las anteriores categorías al consumo de drogas, legales e ilegales, en general.

Las series se han escogido según las recomendaciones elaboradas por revistas digitales especializadas en el ámbito hispanohablante: Fotogramas (2020) y Sensacine (2020). De las series recomendadas por ambas revistas se han escogido las que han obtenido una mayor puntuación popular en Rotten Tomatoes (s/a), es decir, un 50\% mayor de tres estrellas. En total, la muestra ha resultado en siete series de televisión: Elite (Netflix: 2018-En curso), Chilling Adventures of Sabrina (Las escalofriantes aventuras de Sabrina, Netflix: 2018-2020), Sex Education (Netflix: 2019-En curso), 13 Reasons Why (Por trece razones, Netflix: 2017-2020), I Am Not Okay with This (Esta Mierda me supera, Netflix: 2020), Riverdale (The WC: 2017-En curso) y Euphoria (HBO: 2019-En curso).

En definitiva, la ficha de análisis con las variables y las categorías asociadas resultaría de la siguiente forma:

Tabla 1. Ficha de análisis relaciones sexuales

\begin{tabular}{|c|c|c|c|c|c|}
\hline Sexo & Hombre & Mujer & & & \\
\hline $\begin{array}{l}\text { Protección en } \\
\text { relaciones } \\
\text { sexuales }\end{array}$ & Sí & No & & & \\
\hline $\begin{array}{l}\text { Relación } \\
\text { personaje }\end{array}$ & $\begin{array}{l}\text { Pareja } \\
\text { estable }\end{array}$ & $\begin{array}{l}\text { Relación } \\
\text { temporal }\end{array}$ & & & \\
\hline $\begin{array}{l}\text { Relaciones } \\
\text { sexuales }\end{array}$ & $\begin{array}{c}\text { Con } \\
\text { penetración }\end{array}$ & Sexo oral & Masturbación & & \\
\hline Consecuencias & Placer & $\begin{array}{c}\text { Unión con la } \\
\text { pareja }\end{array}$ & Rechazo social & $\begin{array}{c}\text { Embarazo } \\
\text { no deseado }\end{array}$ & ETS \\
\hline
\end{tabular}

Fuente: elaboración propia. Adaptación de Aubrey (2004), Russell, Russell y Grube (2009), Wright (2009), Reñones, Valdivieso López y Victoria Arijaa (2011) y Van Damme (2010). 
Tabla 2. Ficha de análisis consumo estupefacientes

\begin{tabular}{|l|c|c|c|}
\hline $\begin{array}{c}\text { Consecuencias } \\
\text { consumo de } \\
\text { drogas (legales e } \\
\text { ilegales) }\end{array}$ & $\begin{array}{c}\text { En el día a día } \\
\text { rutinario }\end{array}$ & Diversión & $\begin{array}{c}\text { Expresar } \\
\text { sentimientos }\end{array}$ \\
\hline $\begin{array}{l}\text { Desinhibición, } \\
\text { Relajación }\end{array}$ & Habilidades sociales & $\begin{array}{c}\text { Relaciones } \\
\text { sexuales lamentables }\end{array}$ & Adicción \\
\hline Accidente & Muerte & Indigestión & \multicolumn{1}{|l}{} \\
\cline { 1 - 3 } & &
\end{tabular}

Fuente: elaboración propia. Adaptación de Aubrey (2004), Russell, Russell y Grube (2009), Wright (2009),

Reñones, Valdivieso López y Victoria Arijaa (2011) y Van Damme (2010).

Antes de la codificación de las series expuestas, las narrativas han sido desfragmentadas en escenas, siendo estas definidas como unidades de significado en el que uno o varios personajes comparten tiempo y espacio (Casetti y di Chio, 2010). A modo de ilustración, se expone a continuación una muestra de la fragmentación de una unidad de análisis:

Tabla 3. Ejemplo codificación

\begin{tabular}{|c|c|c|c|}
\hline Numeración & $\begin{array}{l}\text { Código } \\
\text { de serie }\end{array}$ & $\begin{array}{l}\text { Temporada } \\
\text { y capítulo }\end{array}$ & Escena \\
\hline 46 & 1 & $1 \times 01$ & $34: 04-39: 12$ \\
\hline
\end{tabular}

Fuente: elaboración propia.

En último lugar se ha dejado espacio a una última variable de corte cualitativo que permitiese dejar constancia de cuestiones reseñables en el apartado de resultados. De este modo se parte de la complementariedad de la metodología cualitativa para exponer datos con una mayor profundidad.

Para la fiabilidad (Krippendorff, 1990) de la plantilla de análisis se ha realizado un test de intracodificador, donde el autor ha codificado el $10 \%$ de la muestra dos veces, dejando un espacio de tiempo de quince días entre ambas codificaciones. El resultado coincidente fue del 99,2\%. El acuerdo, según la fórmula empleada por Holsti (1968), debe superar el 90\% de coincidencia entre ambas codificaciones; por lo tanto, la plantilla diseñada superó la prueba de fiabilidad. 


\subsection{Corpus}

La muestra seleccionada corresponde al total de escenas de los episodios que configuran la primera temporada de cada serie. Con el fin de facilitar la ilustración de los resultados, detallaremos a continuación la sinopsis de cada una de las series analizadas:

a) Élite: Las Encinas es el colegio más exclusivo de España, en el cual estudian los hijos de la élite y donde acaban de ser admitidos tres jóvenes de clase baja, procedentes de un colegio público en ruinas. Las diferencias entre los jóvenes de las distintas clases sociales marcarán las tramas de la serie.

b) Chilling Adventures of Sabrina: Sabrina Spellman debe enfrentar su naturaleza como mitad bruja y mitad mortal. En el enfrentamiento deberá equilibrar su naturaleza dual para proteger de las fuerzas oscuras a su familia, a sus amigos y al mundo que habitan los humanos.

c) Sex Education: Otis Milburn es un adolescente introvertido que mantiene una relación atípica con su madre, quien ejerce como terapeuta sexual y se expresa sin complejos sobre temas íntimos considerados tabús. A pesar de los inconvenientes que le haya podido producir, la educación sexual que Otis ha recibido dista de la recibida por sus compañeros, haciendo que estos le tomen como referente. De este modo, Otis y su amiga Maeve inician una consulta sexual en el instituto para ayudar a sus compañeros.

d) 13 Reasons Why: Clay Jensen comienza a escuchar las cintas grabadas por la fallecida Hannah Baker, una compañera de clase que se suicidó debido a sus problemas en el instituto. En las cintas, Hannah explica a trece personas la forma en que precipitaron su muerte, dando trece razones para explicar por qué se quitó la vida.

e) I Am Not Okay with This: Syd se enfrenta al caos del instituto, los dramas familiares y el amor no correspondido, todo combinado con una sucesión de eventos paranormales que no puede controlar.

f) Riverdale: con el nombre de la misma ciudad, la serie explora los misterios de una comunidad aparentemente idílica, pero repleta de secretos. Los jóvenes adolescentes protagonistas tienen que lidiar con los problemas típicos de la adolescencia mientras aprenden del mundo de los adultos.

g) Euphoria: la serie sigue a un grupo de estudiantes de secundaria que experimentan con las relaciones sexuales, las drogas, el sexo, el amor y la amistad. 


\section{Resultados}

A continuación, procedemos a exponer por bloques temáticos los resultados obtenidos tras la explotación de los datos de las series de adolescentes. Con el fin de ilustrar los resultados, los datos cuantitativos se han ejemplificado con las escenas y tramas más significativas de las series en cuestión.

\subsection{Las relaciones sexuales en las series de adolescentes}

En general, en las series objeto de estudio el sexo no es un tema tabú y los jóvenes, tanto los hombres como las mujeres, disfrutan de una vida sexual sin tapujos; por ejemplo, en Sex Education se trata la masturbación femenina y se anima a las chicas a practicarla para mantener relaciones sexuales más satisfactorias. Las series en las que los adolescentes tienen más relaciones sexuales son Sex Education (31,75\%), Élite (26,98\%), Euphoria (15,87\%) y 13 Reasons Why $(14,29 \%)$. Al contrario, las series con una menor aparición de escenas sexuales son Riverdale (4,76\%), I Am Not Okay with This (3,17\%) y Chilling Adventures of Sabrina (3,17\%). Es más, es característico que en series como Sex Education, Euphoria y I Am Not Okay with This el tabú sea conservar la virginidad, lo que lleva a los jóvenes a experimentar primeras relaciones insatisfactorias con el fin de tener sexo por primera vez. Por otro lado, que todos los personajes traten con desenvoltura las temáticas sexuales no es equivalente a que posean relaciones sanas y seguras. En este sentido, uno de los resultados más concluyentes es la escasa utilización de medidas anticonceptivas:

Figura 1. Uso de preservativo

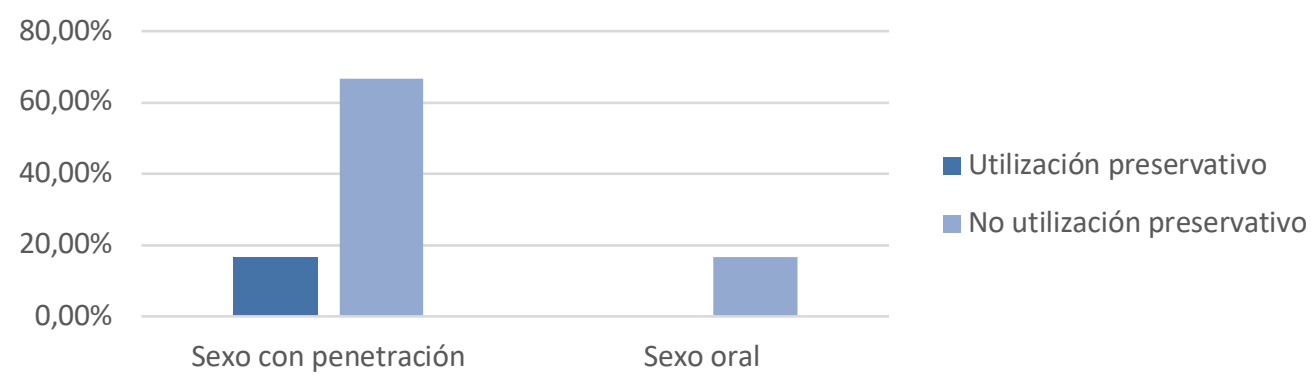

Fuente: elaboración propia.

Así, de todas las escenas en las que algún personaje ha practicado el sexo oral ninguno ha utilizado o mencionado la necesidad de utilizar protección. De igual forma, en las relaciones sexuales con penetración solo un $16,67 \%$ ha uti- 
lizado un preservativo, mientras que un $66,67 \%$ no ha utilizado ninguna medida de protección. Aunque dicho comportamiento sea algo extendido entre ambos sexos, sí que es cierto que las mujeres han sido las que más han mencionado la necesidad de utilizar protección. Sin embargo, dicha responsabilidad solo ha significado un $15 \%$ del total. Cabe mencionar que un $65,75 \%$ de las relaciones sexuales (sexo oral y con penetración) se han producido entre parejas esporádicas, por lo que la utilización de preservativo es esencial. Entre las series que lanzan un mensaje más peligroso se encuentra Élite, en la cual una chica con VIH, Marina, mantiene relaciones sexuales sin protección con dos chicos (en un caso sexo con penetración sin protección y en otro sexo oral, a ella, sin protección). Aunque Marina explica posteriormente que su carga viral es indetectable, y por lo tanto no transmite la enfermedad, el mensaje final puede transmitir una sensación de falsa seguridad a los jóvenes porque, aunque sea cierto que con la medicación existen muy pocos riesgos de transmisión (Capote, Duquernes y Cancio Santander, 2017), la recomendación con parejas esporádicas es siempre utilizar protección. Sí que es laudatorio que el personaje con VIH no haya sido estigmatizado y que finalmente iniciara una relación sentimental con uno de los chicos, quien se enamora de ella y la apoya cuando se queda embarazada de imprevisto.

Figura 2. Exposición relaciones sexuales

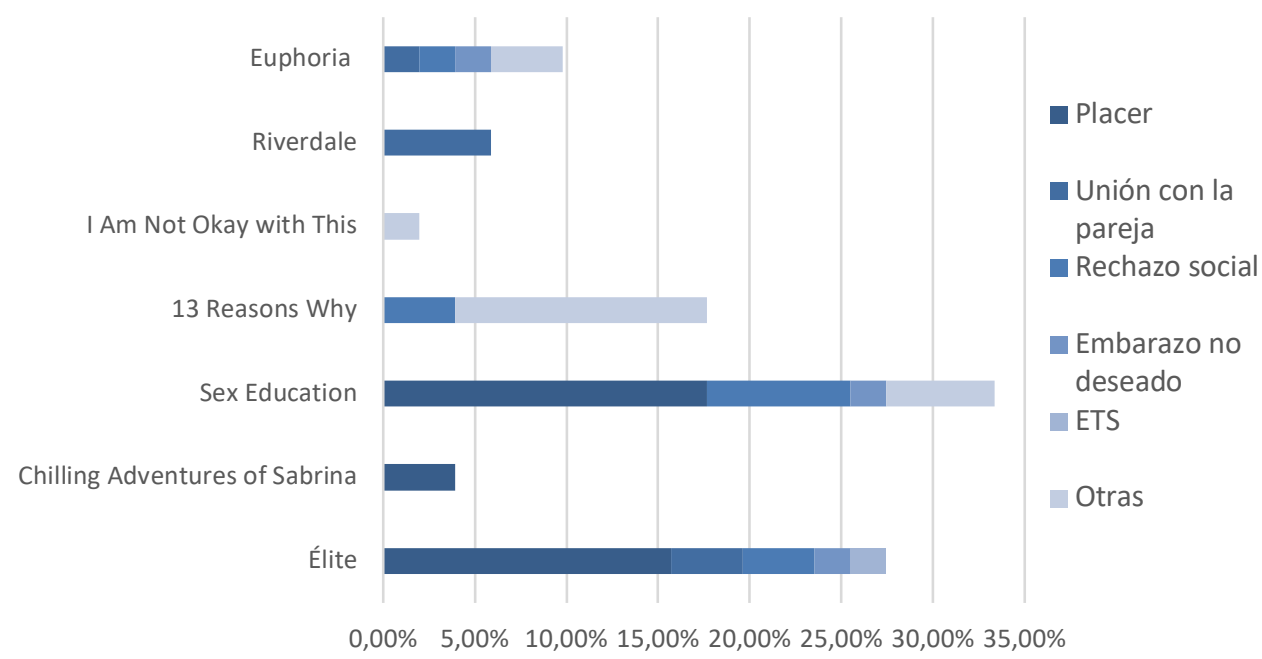

Fuente: elaboración propia. 
La ausencia de medidas de protección se ha traducido en algunos casos de embarazos indeseados (Sex Education; Elite; Euphoria) o de enfermedades de transmisión sexual (Sex Education; Elite). No obstante, en general las relaciones sexuales en las series analizadas se caracterizan por la ausencia de consecuencias: los jóvenes pueden disfrutar del sexo sin protección y sin efectos adversos. De hecho, como se ilustra a continuación, las relaciones sexuales sin protección se caracterizan por su disfrute sin más:

Las series donde han aparecido más problemáticas sociales relacionadas con el sexo son Sex Education (7,84\%), 13 Reasons Why (3,92\%), Élite (3,92\%) y Euphoria (1,92\%). Las consecuencias negativas son, en su totalidad, experimentadas por las chicas. Además del embarazo no deseado y las enfermedades de transmisión sexual, la mayoría de las consecuencias negativas relacionadas con las relaciones sexuales se encuentran conectadas con la vergüenza social; sobre todo, el acoso a las jóvenes por disfrutar de su sexualidad. Por ejemplo, Kat Hernández en Euphoria es una chica que siente presión por perder la virginidad, lo cual consigue en una salvaje fiesta organizada por el chico más popular del instituto. Sin embargo, el orgullo por la recién adquirida experiencia se convierte en vergüenza al descubrir al día siguiente que el chico le grabó un video sin su consentimiento y que lo ha difundido por el instituto. De hecho, en la serie se demuestra que entre los chicos populares es común difundir videos sexuales de las chicas sin su consentimiento y humillarlas; es el caso también de Cassie Howard, quien intenta borrar con vergüenza su pasado. Es de destacar que la serie, a través de los diálogos de los personajes, enfoca la culpa de dicha práctica abusiva a los hostigadores: «cuando estás por alguien pasas fotos, la culpa es de ellos por pasarlas» (Rue, 1x1). Además, es característico que el chico más popular del instituto (y el principal hostigador de las malas prácticas) sea Nate Jacobs, un hombre con problemas de agresividad que mantiene una relación de control y abuso con Maddy Pérez, de quien le atrajo su aspecto virginal. Además de en Euphoria, la difusión de videos sexuales sin consentimiento también se visualiza en Sex Education, aunque en este caso la amenaza se soluciona con un ejercicio de sororidad entre compañeras. En este sentido, ante las fotos difundidas de la vagina de Ruby, todas las chicas se levantan en el auditorio para decir que la foto es suya.

Uno de los casos más sensibles sobre el consentimiento se produce en 13 Reasons Why, en la que su fallecida protagonista, Hannah Baker, es testigo y víctima de dos violaciones perpetradas por Bryce Walker, uno de los populares del instituto. En el primer caso es testigo de la violación por parte de este a su amiga Jessica, de quien se aprovecha mientras se encuentra inconsciente por la in- 
gesta de alcohol. En el segundo caso, Hannah es violentamente agredida durante una fiesta y se muestra gráficamente cómo el intento de lucha por la resistencia se torna en un estado inerte de la víctima, a quien se le despoja de su humanidad. En este sentido, en varias escenas anteriores se demuestra que Bryce Walker no considera a las mujeres como seres humanos con derechos propios (como, por ejemplo, al tocar el trasero de Hannah en el tercer capítulo exponiendo que estaba a mano). Las dos violaciones tienen consecuencias para sus víctimas: Jessica sospecha que abusaron de ella y cae en una espiral de alcoholismo, mientras que Hannah se suicida poco después. La objetivación sexual de las mujeres también se discute en Euphoria, en Élite y en Sex Education. En Euphoria, además de los casos ya mencionados, podemos señalar, por ejemplo, el recuerdo de la protagonista de Nate Jacobs metiéndole sus dedos en la vagina sin su consentimiento en el transcurso de una fiesta. En Sex Education, Maeve Wiley recuerda entristecida cómo un chico se inventó un rumor sexual sobre ella y el aislamiento social que le impusieron sus compañeros de instituto por aquello. En Élite es una chica quien instrumentaliza el sexo: Lucrecia Montesinos quiere que su compañero, Guzmán, seduzca a Nadia Shana y evidenciarla en público.

\subsection{Alcohol, tabaco y drogas ilegales en series de adolescentes}

$\mathrm{Al}$ igual que el sexo, en las series objeto de estudio las drogas, en general, no son un tema tabú y los jóvenes, tanto los hombres como las mujeres, experimentan con ellas. El alcohol es el consumo que se encuentra más normalizado en las series, siendo Élite (44,06\%), Sex Education (17,82\%), Euphoria $(17,80 \%)$ y 13 Reasons Why (14,36\%) donde los jóvenes aparecen más consumiendo cerveza o bebidas de alta graduación. Las series donde los jóvenes apenas consumen alcohol son Chilling Adventures of Sabrina y Riverdale. Por su parte, el tabaco se encuentra menos extendido, y son los jóvenes considerados como problemáticos quienes tienen el hábito de fumar: Cristian y Nano en Élite, o Maeve Wiley en Sex Education. Como se muestra en la gráfica a continuación, la bebida se suele encontrar contextualizada en ambientes festivos $(44,12 \%)$, donde los jóvenes beben para divertirse y con apenas consecuencias nefastas: 
Figura 3. Consecuencias consumo de drogas legales

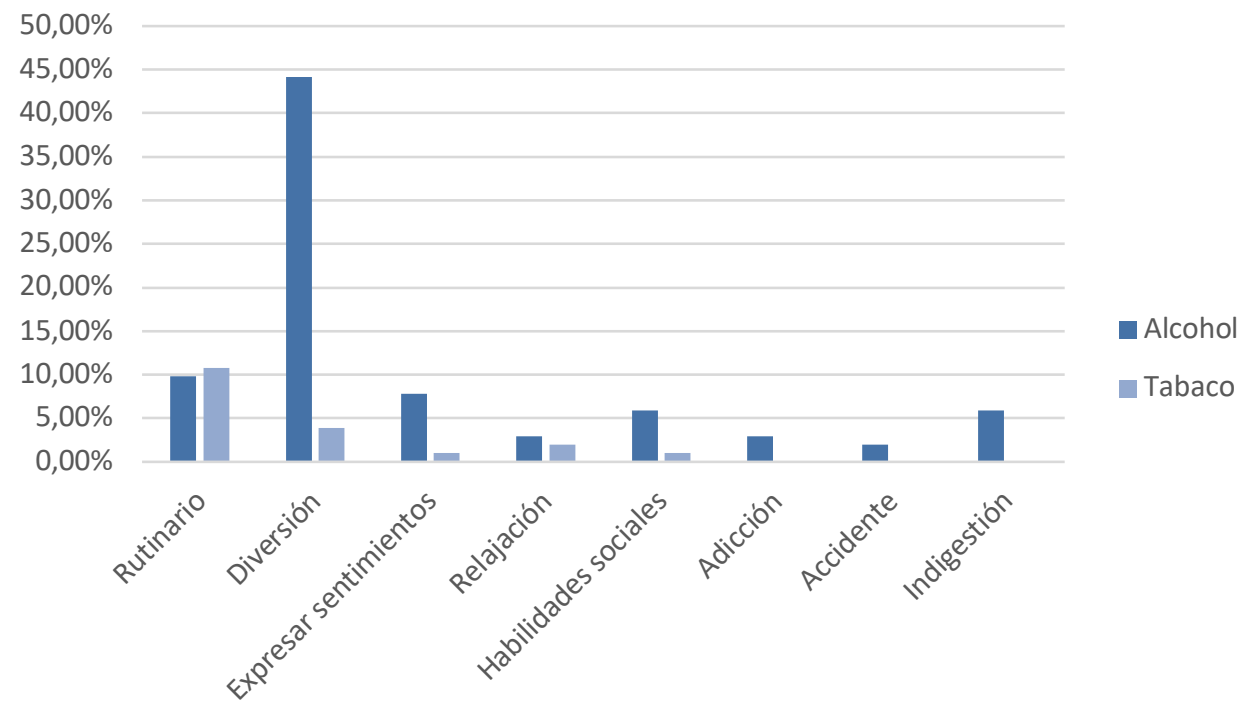

Fuente: elaboración propia.

La adicción solo aparece en 13 Reasons Why en el ya mencionado caso de Jessica, quien cae en el consumo descontrolado de alcohol cuando sospecha que ha sido víctima de una violación mientras se encontraba ebria. En la misma serie se produce un accidente por conducir bajo los efectos del alcohol, cuando Sheri Holland derriba una señal de stop y provoca un mortal accidente. Aparte de los anteriores casos, el consumo de alcohol no supone una actividad de ocio peligrosa para los jóvenes, quienes se embriagan para divertirse, para conocer nuevas amistades o para declararse a una persona. Respecto al consumo por sexos, se destaca un mayor consumo entre los hombres $(47,52 \%)$ que las mujeres $(33,66 \%)$ : 
Figura 4. Consumo drogas legales por sexo

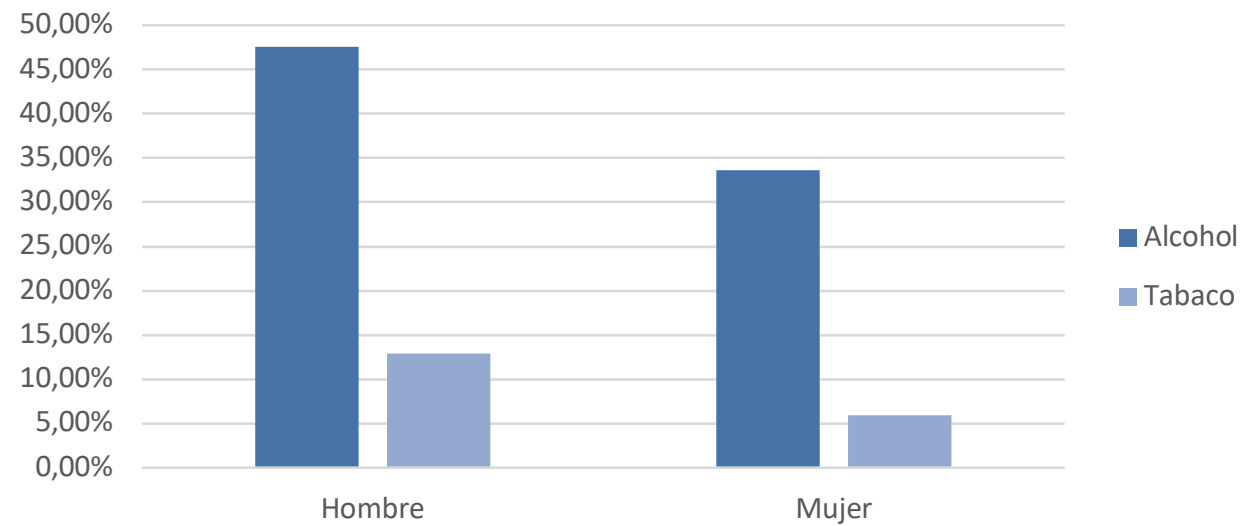

Fuente: elaboración propia.

Por su parte, las drogas ilegales hacen un menor grado de aparición. En primer lugar, las drogas naturales como la marihuana o el hachís aparecen más en Elite (44,06\%), Sex Education (17,82\%), Euphoria (14,85\%) y 13 Reasons Why (14,81\%). Los perfiles más habituales de consumo son chicos en contextos rutinarios, como después de las clases o en reuniones en los barrios. Las drogas sintéticas son consumidas en mayor medida en Euphoria (58,53\%), en Élite (15,14\%) y en Sex Education (5,86\%), mientras que no aparecen en Chilling Adventures of Sabrina, Riverdale y I Am Not Okay with This. En Riverdale sí que se menciona el narcotráfico, pero los protagonistas no sienten la tentación de probarlo y se asocia dicha actividad con el asesinato de Jason Blossom, uno de los chicos populares del instituto. De hecho, el consumo de drogas sintéticas es el que asocia en general a mayores consecuencias negativas. En Élite contaminan el ponche sin alcohol con pastillas sintéticas y Nadia Shana consume sin ser consciente, provocando en ella un estado de desinhibición que la tienta a seducir a Guzmán. En Sex Education un chico casi provoca un accidente mortal al consumir las pastillas que le vendió un camello de barrio. Por último, en Euphoria hace aparición Rue Bennet, una chica con problemas de adicción que ha estado al borde de la muerte por el consumo de drogas. No obstante, aunque sean predominantes las consecuencias negativas, en el consumo de drogas ilegales también aparece un considerable porcentaje de consecuencias positivas como la diversión (14,29\%) o la relajación $(11,90 \%)$. En resumen, las consecuencias del consumo de drogas en las series analizadas se ilustran con la siguiente gráfica: 
Figura 5. Consecuencias consumo de estupefacientes

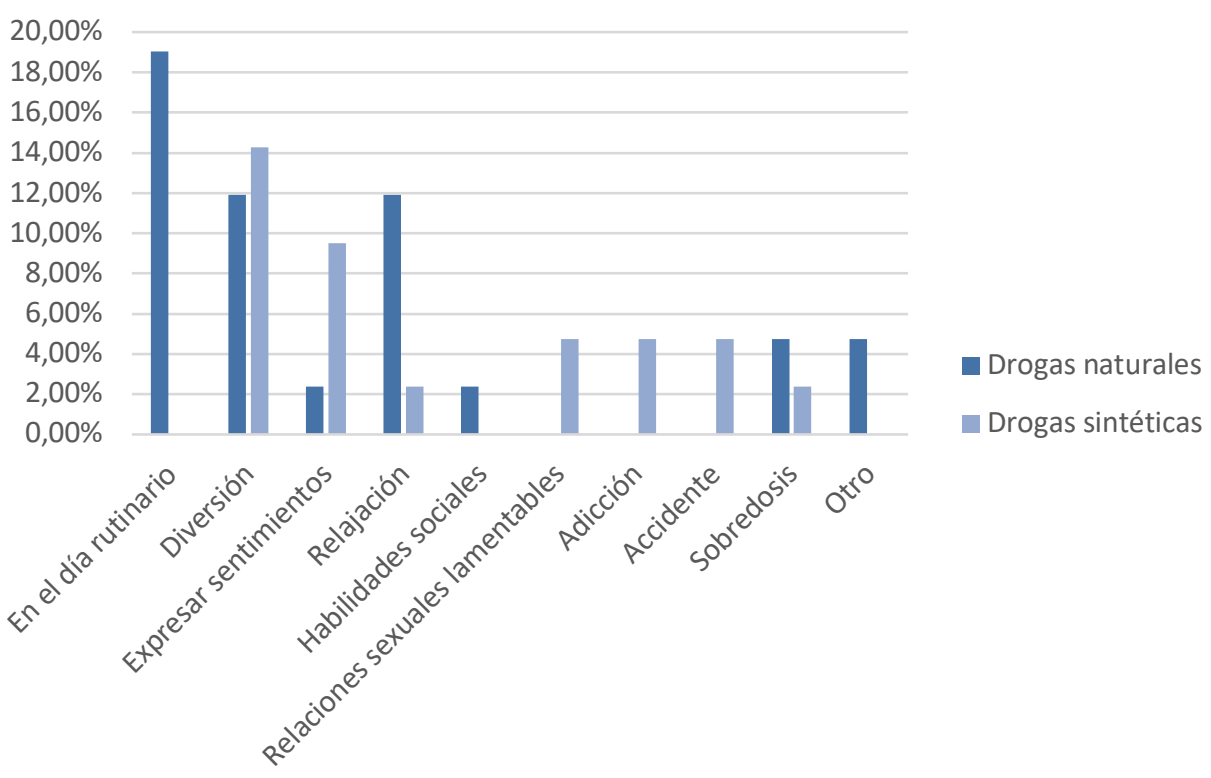

Fuente: elaboración propia.

\section{Discusión y conclusión}

Los datos recabados afirman la recurrencia de temas sexuales y de drogas en las series dirigidas a adolescentes. No obstante, cabe subrayar que dichas temáticas hacen un menor grado de aparición en las series enmarcadas en el género fantástico; por ejemplo, en The chilling adventures of sabrina las relaciones sexuales entre los personajes ocurren muy esporádicamente, el consumo de alcohol es muy limitado y las drogas ilegales no hacen aparición.

En las series analizadas, ligadas a la experimentación sexual y el ocio se encuentran las temáticas de aceptación personal y la amistad, ya apuntadas en el trabajo de García-Muñoz y Fedele (2011). En las narrativas estudiadas los adolescentes navegan entre distintas relaciones y ambientes para encontrar su propia identidad: «Los adolescentes están buscando su propia identidad sexual, y las relaciones les hacen reflexionar sobre sí mismos y sobre quién quieren ser» (Van Damme, 2010: 86) ${ }^{4}$. Respecto al sexo, y al igual que en las series de la década de los noventa como Saved by the Bell (NBC: 1989-1992), la práctica sexual más representada en las series de adolescentes ha sido el coito completo

\footnotetext{
${ }^{4}$ The teenagers are searching for their own sexual identities, and relationships make them reflect about themselves and who or how they want to be.
} 
(Crespo, 2005). En concordancia con otros estudios sobre series de adolescentes (Kunkel et. al. 2001; Sprafkin y Silverman, 1981; Tuglio, 1998; Aubrey, 2004; Hust, Brown y L'Engle, 2008; L'Engle, Brown, y Kenneavy, 2006; Wright, 2009), los personajes sexualmente activos rara vez toman precauciones contra el embarazo o las enfermedades de transmisión sexual. Asimismo, en pocas ocasiones experimentan consecuencias negativas como resultado de sus imprudencias sexuales y, cuando toman medidas de protección, ellas son las únicas responsables de la anticoncepción (Gavin, 2001; Batchelor et al., 2004; Van Damme y Van Bauwel, 2013). Cabe apuntar que, siguiendo la tendencia de otras teen series (Aubrey, 2004; Ortiz y Brooks, 2014), en los casos en los que aparecen efectos adversos negativos, son las chicas quienes tienen una mayor probabilidad de sufrir las consecuencias.

No obstante, sí que hay una diferencia significativa en las series aquí examinadas y otras teen series como Beverly Hills 90210 (1990-2000), My So-Called Life (1994-1995), Dawson's Creek (1998-2003), Freaks and Geeks (19992000), Smallville (2001-2011), The O.C. (2003-2007) o Gossip Girl (20072012). En dichas series cuando se produce una agresión sexual la figura del perpetrador queda invisibilizada, «La violencia sexual, por tanto, se convierte en algo que tiene que ver con las mujeres, no en algo cometido por los hombres. Los perpetradores son presentados como figuras sombrías y desaparecen poco después de cometer el acto violento para no volver jamás» (Berridge, 2011: 478) ${ }^{5}$. Al contrario, en 13 Reasons Why o en Euphoria los agresores son personajes recurrentes y el foco de la narrativa recae en sus actitudes nocivas. Además, en las series de este estudio, al contrario que en otras con la misma temática (Aubrey, 2004; Bindig, 2008; Ortiz y Brooks, 2014), no se tiende a caracterizar a las chicas sexualmente activas como 'malas chicas'. De hecho, cuando son degradadas como objetos sexuales la posición es discutida y rebatida por las protagonistas, como en Euphoria, donde se señala el papel nocivo de la industria pornográfica. De este modo, al igual que los resultados del trabajo de Mateos-Pérez (2021), las series analizadas toman consciencia de ciertas problemáticas que acechan a los jóvenes:

Lo relevante de la ficción es que trata de responder a través de sus personajes y sus valores - de amistad, tolerancia, generosidad- de forma integradora, tratando de que el espectador tome conciencia de las problemáticas y ofreciéndole recursos para afrontar e imponerse sobre estas (Mateos-Pérez, 2021: 151).

\footnotetext{
5Sexual violence, then, becomes something that is about women, not something that is done by men. Male perpetrators of sexual violence are presented as shadowy figures in these episodes, disappearing shortly after committing the violent act, never to return.
} 
Aparte, atrás queda la concepción de que la perdida de la virginidad es un asunto social para los chicos y moral para las chicas (Kelly, 2010; Crowley Webber, 2019). En Sex Education, Euphoria, Élite o I'm not Okay with This el sexo es para todos un tema social: todo su entorno ha practicado sexo y temen quedarse rezagados, «La metáfora de la pérdida de la virginidad como rito de paso sugiere que la pérdida de la virginidad es un proceso vital para el desarrollo de la persona» (Kelly, 2010: 486) ${ }^{6}$.

En lo concerniente al consumo del alcohol, tabaco y otras drogas encontramos que su consumo se encuentra normalizado y que, por lo general, no conllevan consecuencias negativas (a excepción de las drogas sintéticas). La práctica que hace un menor número de apariciones es el hábito de fumar que, siguiendo la dinámica de series como Física o Química, es asociado a los personajes rebeldes o "malotes" (Reñones, Valdivieso López y Victoria Arijaa, 2011) como Maeve Willis de Sex Education o Christian Varela de Élite. Por su parte, el consumo del alcohol es el más habitual en las escenas de ocio, siendo Élite (44,06\%), Sex Education (17,82\%), Euphoria (17,80\%) y 13 Reasons Why $(14,36 \%)$ donde los jóvenes aparecen más consumiendo cerveza o bebidas de alta graduación. Dicha tendencia ya ha sido encontrada en anteriores estudios sobre series de adolescentes (Russell, Russell y Grube, 2009; Chapoton, Werlen y Denois 2019) donde, al igual que en las series aquí examinadas, apenas se aprecian consecuencias negativas por su consumo. La excepción la podemos encontrar con 13 Reasons Why, donde el alcohol ha provocado un accidente mortal y adicción. De hecho, la mencionada serie ha provocado una amplia discusión académica debido a los temas sensibles que toca, como el bullying, las agresiones sexuales o el suicidio (Raya Bravo, Sánchez-Labella y Durán, 2018; Chesin, Cascardi, Rosselli, Tsang y Jeglic, 2020; Scalvini, 2020; Nesi, Johnson, Altemus, Thibeau, Hunt y Wolff, 2020; Pérez-Martínez, Aparicio Vinacua,y Rodríguez-González, 2020).

En síntesis, los protagonistas adolescentes de las ficciones televisivas tienen una vida sexual activa y consumen alcohol regularmente. El tabaco es un hábito menos común y se encuentra asociado con personajes rebeldes, mientras que las drogas naturales como la marihuana se consideran inofensivas. Por su parte, las drogas sintéticas acarrean más problemas personales, aunque su consumo también se asocia a la diversión. Los jóvenes, tanto los hombres como las mujeres, poseen una concepción del tiempo a corto plazo y no suelen tomar precauciones ante el riesgo. De esta forma, los adolescentes no han utilizado

\footnotetext{
${ }^{6}$ The metaphor of virginity loss as a rite of passage suggests that virginity loss is one step in the process of growing up and one moment in the sexual life course.
} 
medidas anticonceptivas en una gran proporción de las escenas sexuales analizadas. Dichos actos de los jóvenes apenas tienen consecuencias negativas, pero cuando se han dado se ha tratado el tema con sensibilidad y profundidad (embarazos no deseados, enfermedades transmisión sexual, accidentes...). Además, es característico que el sexo en las mujeres ya no sea una cuestión moral, que se superen tabúes sexuales como la masturbación femenina o se cuestione el patriarcado poniendo el foco en los comportamientos machistas. No obstante, y en línea con otras investigaciones (Gavin, 2001; Batchelor et al., 2004; Van Damme y Van Bauwel, 2013; Lacalle, 2013), lo anterior se contrapone con representaciones estereotípicas tradicionales. Así, cuando se producen consecuencias negativas por el sexo quienes sufren los efectos son las chicas y, en las pocas ocasiones en las que se utiliza protección, son ellas quienes recuerdan la necesidad de utilizar medidas anticonceptivas.

A modo de conclusión, en las series dirigidas a adolescentes analizadas se dan simultáneamente la ruptura de ciertos estereotipos y el mantenimiento de otros. En la ficción, al igual que en la realidad social, los cambios actitudinales se producen gradualmente $\mathrm{y}$, como hemos podido observar, ciertas costumbres o creencias oponen una considerable resistencia al cambio. No obstante, cuando los personajes de las teen series rompen la narrativa tradicional, cabe resaltar que los temas son tratados con complejidad y en profundidad. Así, por ejemplo, es laudatorio que en dichas series la violencia ejercida contra la mujer sea objeto de un serio debate y que la culpa no recaiga sobre la víctima, sino sobre el instigador. En línea con los resultados obtenidos, sería interesante plantear como futura línea de investigación el conocer la interpretación de dichas narrativas por los adolescentes.

\section{Referencias bibliográficas}

ALBERO, M. (2005). Violencia, sexo y televisión: la mirada adolescente, en Quaderns del CAC, 22, 81-90. https:// bit.ly/3aNURWI

ARNETT, J., JEFFREY, R. y OFFER, D. (1995). Beyond effects: Adolescents as active media users, en Journal of Youth and Adolescence, 24(5), 511-51. doi.org/10.1007/BF01537053

AuBREY, J. (2004). Sex and punishment: An examination of sexual consequences and the sexual double standard in teen programming, en Sex Roles, 50 (7/8) 505-514.

doi.org/10.1023/B:SERS.0000023070.87195.07

Austin, E. W. y MEILI, H. (1994). Effects of Interpretations of Televised Alcohol Portrayals on Children's Alcohol Beliefs, en Journal of Broadcasting and Electronic Media, 38(4), 417-435. doi.org/10.1080/08838159409364276 
BANKS, M. (2004). A Boy for All Planets: Roswell, Smallville and the Teen Male Melodrama, en DAVIS, GLYN y DiCKINSON, Kay (Ed.), Teen TV. Genre, Consumption and Identity (pp. 17-28), London: British Film Institute.

BATCHELOR, S. y KITZINGER, J. (2004). Representing young people's sexuality in the "youth" media, en Health Education Research, 19(6), 669-676. doi.org/10.1093/her/cyg082

BERRIDGE, S. (2011). Personal Problems and Women's Issues, en Feminist Media Studies, 11(4), 467-481. doi.org/10.1080/14680777.2011.555967

BRoWn, J. D., STEELE, J. R. y WALSCH-ChILDERS, K. (2002). Sexual teens, sexual media. Investigating media's influence on adolescent sexuality. New York, NY: Lawrence Erlbaum Associates.

CASTELLÓ, E. (2010). Dramatizing proximity: Cultural and social discourses in soap operas from production to reception. European Journal of Cultural Studies, 13(2), 207-223. doi.org/10.1177/1367549409352274

MATOS CAPOTE, M. D., DuQUERNES, A. L. y CANCIO SANTANDER, J. M. (2017). Reflexiones acerca de las ITS, el VIH y el SIDA. Revista Márgenes, 6(1), 8395. https: //cutt.ly/ERDoFvF

Chesin, M., CASCARSI, M., Rosselli, M., TSANG, W. y JeGliC, E. L. (2020). Knowledge of Suicide Risk Factors, But Not Suicide Ideation Severity, Is Greater Among College Students Who Viewed 13 Reasons Why, en Journal of American College Health, 68(6), 644-649.

\section{doi.org/10.1080/07448481.2019.1586713}

Christensen, P. G., HENRIKSEN, L., y DonALD, F. R. (2000). Substance Use in Popular Prime-Time Television. Washington, DC: Office of National Drug Control Policy.

CRESPO, M. (2005). Mensajes y modelos televisivos para los adolescentes: estudio base para un análisis sistemático del contenido sexual de las series de televisión, en Doxa.Comunicación, 3, 187-213.

\section{doi.org/10.31921/doxacom.n3a11}

DHAENENS, F. (2012) Reading Gays on the Small Screen, Javnost - The Public, 19(4), 57-72. doi.org/10.1080/13183222.2012.11009096

EYAL, K., KUNKEL, D., BIELY, E. N. y FINNERTY, K. (2007). Sexual socialisation messages on television programs most popular among teens, en Journal of Broadcasting \& Electronic Media, vol. 51, num. 2, pp. 316-336.

FALCÓN, L. y DíAZ-AGUADO, M. J. (2014). Adolescent Students as Media Fictional Characters., en Comunicar, 21(42), 147-155. doi.org/10.3916/C42-2014-14

FEASY, R. (2006). Charmed: Why Teen Television Appeals to Women, en Journal of Popular Film and Television, 34(1), 2-9. doi.org/10.3200/JPFT.34.1.2-9 
FEDELE, M. y GARCÍA-MuÑOZ, N. (2010). El consumo adolescente de la ficción seriada, en Vivat Academia, 111, 48-65.

doi.org/10.15178/va.2010.111.47-64

GUNTER, B. (1995). Television and gender representation. London: John Libbey \& Company.

HunDLEY, H. L. (1995). The Naturalization of Beer in Cheers, en Journal of Broadcast and Electronic Media, 39(3),350-359.

doi.org/10.1080/08838159509364311

HUST, S., BROWN, J. y L'ENGLE, K. (2008). Boys will be boys and girls will be better prepared: An analysis of the rare sexual health messages in young adolescents' media, en Mass Communication and Society, 11(1), 3-23. doi.org/10.1080/15205430701668139

JENSEN, K. (1994). Reception as flow: The 'New television viewer' revisited, Cultural Studies, 8(2), 293-305. doi.org/ 10.1080/09502389400490461

KELLNER, D. (1995). Media culture: Cultural studies, identity, and politics between the modern and the post modern. London: Routledge.

KELLY, M. (2010). Virginity loss narratives in "teen drama" television programs, en Journal of Sex Research, 47(5), 479-489. doi.org/10.1080/00224490903132044

KIM, J., SORSOLI, L., CollinS, K., ZYLBERGOLD, B. y SCHOOLER, D. (2007). From sex to sexuality: Exposing the heterosexual script on primetime network television, en Journal of Sex Research, 4(2), 145-157. doi.org/10.1080/00224490701263660

L'ENGLE, K., BRoWn, J. D. y KENNEAVY, K. (2006). The mass media are an important context for adolescents' sexual behavior, en Journal of Adolescent Health, 38(3), 186-192. doi.org/10.1016/j.jadohealth.2005.03.020

LACALLE, C. (Ed.) (2013). Jóvenes y ficción televisiva. Construcción de la identidad y transmedialidad. Editorial UOC: Barcelona.

MaRch CERDÁ, J. C., MARTín BARATO, A., LóPEz Doblas, M., LuQue MARTíN, N. y PRIETo RodríGUEZ, M. A. (2014). La publicidad de bebidas alcohólicas en España y su repercusión en la población adolescente, en Revista española de drogodependencias, 4, 59-76. https:// bit.ly/3FSXSU5

MASANET, M. J. (2016). Pervivencia de los estereotipos de género en los hábitos de consumo mediático de los adolescentes: drama para las chicas y humor para los chicos. Cuadernos.info, 39, 39-53. doi.org/10.7764/cdi.39.1027

MASANET, M. J. y ARAN-RAMSPOTT, S. (2016). The Survival of the Forbidden Love in Television Fiction: 'Romeo and Juliet' in Contemporary Spanish Series for Youth, en Hetsroni, Amir (Ed.), Television and Romance: Studies, Observations and Interpretations (pp.19-38), New York: Nova Science. 
MATEOS-PÉREZ, J. (2021). Modelos de renovación en las series de televisión juveniles de producción española. Estudio de caso de Merlí (TV3, 2015) y Skam España (Movistar, 2018), Doxa. Comunicación, 32, 143-157. doi.org/10.31921/doxacom.n32a7

NAERLAND, T.U. (2020). From pleasure to politics: Five functions of watching TV-series for public connection. European Journal of Communication, 35, 93-107. doi.org/10.1177/0267323119894481

NenI, J., Johnson, S. E., ALteus, M., HunT, J. y WolfF, J. (2020). 13 Reasons Why: Perceptions and Correlates of Media Influence in Psychiatrically Hospitalized Adolescents, en Archives of Suicide Research. doi.org/ 10.1080/13811118.2020.1779155

ORTíz, R. y BRooKS, M. E. (2014). Getting What They Deserve? Consequences of Sexual Expression by Central Characters in Five Popular Television Teen Dramas in the United States, en Journal of Children and Media, 8(1), 40-52. doi.org/10.1080/17482798.2014.863477

OSGBERY, B. (2004). 'So Who's Got Time for Adults!': Femininity, Consumption and the Development of Teen TV - from Gidget to Buffy. En Davis, Glyn y Dickinson, Kay (Eds.), Teen TV. Genre, Consumption and Identity (pp. 7186). London: British Film Institute.

OWEN, S. (1999). Vampires, Postmodernity, and Postfeminism: Buffy the Vampire Slayer, en Journal of Popular Film and Television, 27(2), 24-31. doi.org/10.1080/01956059909602801

PÉREZ-MARTíneZ, V. M., APARICIO VinACUA, B. y RodríGuEZ-GonZÁlEZ, M. D. (2020]. Acoso escolar, violación y suicidio en Twitter: segunda temporada de "por trece razones». Vivat Academia, 153, 137-168. doi.org/10.15178/va.2020.153.137-168

RAYA-BRAVO, I., SÁNCHEZ-LABELLA, I., y DURÁN, V. (2018). La construcción de los perfiles adolescentes en las series de Netflix Por trece razones y Atípico, en Comunicación y Medios, num. 37, 131-143. doi.org/10.5354/0719-1529.2018.48631

REY REÑONES, C., VALDIVIESO LOPEZA, E. y ARIJAA, V. (2012). Publicidad encubierta de tabaco en series de producción española, en Atención Primaria, 44(10), 573-575. doi.org/10.1016/j.aprim.2012.06.005

RuSSELL, C. (2006). Consumer Researchers for Public Health: Insights from Three Government-Funded Programs, en Pechmann, Cornelia y Price, Linda (Eds.), Advances in Consumer Research. Ann Arbor: Association for Consumer Research.

RuSSELL, C, y RuSSELL, D. W. (2009). Alcohol Messages in Prime-Time Television Series, en The Journal of consumer affairs, 43(1), 108-128. doi.org/10.1111/j.1745-6606.2008.01129.x 
RuSSELL, D. W., y RuSSELL, C. (2008). Embedded Alcohol Messages in Television Series: The Interactive Effect of Warnings and Audience Connectedness on Viewers' Alcohol Beliefs, en Journal of Studies on Alcohol and Drugs, 69, 459-467. doi.org/10.15288/jsad.2008.69.459

SCALVINI, M. (2020). 13 Reasons Why: can a TV show about suicide be 'dangerous'? What are the moral obligations of a producer?, en Media, Culture \& Society, 42(7-8), 1564-1574.

doi.org/10.1177/0163443720932502

SignORIELLY, N. (2007). How are Children and Adolescents Portrayed on Prime-time Television, en MAZZARELlA, SHARON (Ed.), 20 Questions about Youth and the Media (pp. 167-178), New York: Peter Lang.

VAN DAMME, E. (2010). Gender and sexual scripts in popular US teen series: A study on the gendered discourses in One Tree Hill and Gossip Girl, en Journal of Catalan Studies, 2(1), 77-92. doi.org/10.1386/cjcs.2.1.77_1

VAN DAMmE, E. y VAN BAUWEL, S. (2013). Sex as Spectacle, en Journal of Children and Media, $7(2), 170-185$. doi.org/10.1080/17482798.2012.673499

WRIGHT, P. J. (2009). Sexual socialization messages in mainstream entertainment mass media: A review and synthesis, en Sexuality \& Culture, 13(4), 181-200. doi.org/10.1007/s12119-009-9050-5 